\title{
The matrix-based description approach for the multistage differential-algebraic processes
}

\author{
Paweł Drạg \\ Department of Control Systems and Mechatronics \\ Wrocław University of Technology \\ Janiszewskiego 11-17, 50-372 Wrocław, Poland \\ Email: pawel.drag@pwr.edu.pl
}

\author{
Krystyn Styczeń \\ Department of Control Systems and Mechatronics, \\ Wrocław University of Technology \\ Janiszewskiego 11-17, 50-372 Wrocław, Poland \\ Email: krystyn.styczen@pwr.edu.pl
}

\begin{abstract}
In the article a new insight into an optimal control problem of the multistage processes has been given. The multistage descriptor processes with differential-algebraic constraints are under considerations. The new representation of the descriptor model has been presented. Moreover, the new structures to represent the differential state variables, algebraic state variables, control function, as well the global parameters have been introduced. The generalized description enables the unified representation of a broad group of the multistage processes with differential-algebraic relations and indicates on the physical interpretation of the process variables.
\end{abstract}

Index Terms-optimal control, descriptor systems, DAE systems, generalized description approach.

\section{INTRODUCTION}

$\mathbf{I}$ N THE article some new aspects of the multistage descriptor control systems and their unified representation have been discussed. The control and optimization of the technological processes with the differential-algebraic constraints have nowadays a great importance [12], [18], [19]. Therefore, one of main aims of the presented considerations is to emphasize the applicability of the obtained theoretical results.

The multistage technological processes are often designed in technology, especially in biotechnology [1], [9], [10], [11], chemical engineering [4], [8], as well as in environmental engineering [13], [14], [15].

The presence of a large number of successive stages is a characteristic feature of the modern technological processes. This reflects a complexity, as well as an arrangement of the designed processes. Therefore, the large number of the state variables, control functions and other process parameters needs a generalized description of the multistage systems. The generalized process description can enable us to unify the new control algorithms design.

The large-scale technological systems should be flexible to change their configurations, as well as to be open for necessary modifications. These requirements indicate a one of the new, unified process description approach, which can enable the process to develop.

To design the methodology, which allows us the integration of the additional stages within the process, is a task that requires the specific theoretical basis preparation. The generalized description methodology is the main result of the carried out considerations.
The rest of the paper has been organized in the following way. In Section II the general optimal control problem with the multistage differential-algebraic constraints has been formulated. In Section III the generalized description approach for the multistage DAE system has been proposed. Then, in Section IV, the generalized description approach has been illustrated with the three-stage chemical process. In Section $\mathrm{V}$ the presented considerations have been concluded.

\section{STATEMENT OF THE OPTIMAL CONTROL PROBLEM}

The origin of the considerations about the multistage descriptor processes analysis are connected with two articles [16] and [17]. The assumptions and algorithms proposed in these articles are treated as the mile stones in control and optimization of the multistage technological processes. The observed progress in the control algorithms has been presented in details in three monographs [2], [3], [5].

In this section the most important features of the optimal control problem with the multistage differential-algebraic constraints have been given.

Assumption 2.1: The multistage process are consisted on the $N$ successive stages, where $N$ is the known number and $N \in \mathcal{N}$.

Assumption 2.2: [6] Each stage can be described by the system of the differential-algebraic equations. The index of the DAEs system is not greater than 1 .

Moreover, to simulate, optimize and control a process, the time range has to be known.

Assumption 2.3: The range of the process time duration is known a priori and

$$
t \in\left[\begin{array}{ll}
t_{0} & t_{F}
\end{array}\right]
$$

According to the Assumptions 2.1 and 2.3, the time domain of the each considered stage can be defined separately.

Definition 2.1: The time domain of the stage number $i=$ $1, \cdots, N$ can be defined as

$$
t^{i} \in\left[\begin{array}{ll}
t_{0}^{i} & t_{F}^{i}
\end{array}\right] .
$$


In the stage number $i$ and during the time domain $t^{i}$ the process is governed by the set of the differential-algebraic equations.

Assumption 2.4: [7] At the stage number $i$, the process is governed by the set of the differential-algebraic relations

$$
\begin{aligned}
\dot{\mathbf{y}}^{i}(t) & =F^{i}\left(\mathbf{y}^{i}(t), \mathbf{z}^{i}(t), \mathbf{u}^{i}(t), \mathbf{p}, t^{i}\right) \\
0 & =G^{i}\left(\mathbf{y}^{i}(t), \mathbf{z}^{i}(t), \mathbf{u}^{i}(t), \mathbf{p}, t^{i}\right)
\end{aligned}
$$

where $i=1, \cdots, N$ is the number of the stage considered, $\mathbf{y}^{i}(t) \in \mathcal{R}^{n_{\mathbf{y}^{i}}}$ is a differential state variable, $\mathbf{z}^{i}(t) \in \mathcal{R}^{n_{\mathbf{z}^{i}}}$ is an algebraic state variable and $\mathbf{u}^{i}(t) \in \mathcal{R}^{n_{\mathbf{u}^{i}}}$ denotes the unknown control function. The independent variable (e.g. time or length of the chemical reactor) is denoted as $t \in \mathcal{R}$.

Definition 2.2: The relations in (3) are defined as follows

$$
F^{i}: \mathcal{R}^{\mathbf{y}^{i}} \times \mathcal{R}^{\mathbf{z}^{i}} \times \mathcal{R}^{\mathbf{u}^{i}} \times \mathcal{R}^{\mathbf{p}} \times \mathcal{R} \rightarrow \mathcal{R}^{\mathbf{y}^{i}}
$$

and

$$
G^{i}: \mathcal{R}^{\mathbf{y}^{i}} \times \mathcal{R}^{\mathbf{z}^{i}} \times \mathcal{R}^{\mathbf{u}^{i}} \times \mathcal{R}^{\mathbf{p}} \times \mathcal{R} \rightarrow \mathcal{R}^{\mathbf{z}^{i}}
$$

The minimized process performance index, which can be treated as the measure of the control quality, is defined as follows

$$
\begin{aligned}
& \min _{\mathbf{u}^{i}(t), i=1, \cdots, N} Q \\
& =\sum_{i=1}^{N} \int_{t_{0}^{i}}^{t^{i}} \mathcal{L}\left(\mathbf{y}^{i}\left(t^{i}\right), \mathbf{z}^{i}\left(t^{i}\right), \mathbf{u}^{i}\left(t^{i}\right), \mathbf{p}, t^{i}\right) d t \\
& +\mathcal{E}\left(\mathbf{y}^{N}\left(t_{F}^{N}\right), \mathbf{z}^{N}\left(t_{F}^{N}\right), t_{F}^{N}\right) .
\end{aligned}
$$

\section{THE GENERALIZED DESCRIPTION APPROACH}

In this section we would like to propose the new generalized description approach for the multistage differential-algebraic processes. The presented methodology extends the proposition from the article [16].

Definition 3.1: The particular differential state vector for the $i$-th stage $\mathbf{y}^{i}$ is consisted from all the differential state variables in the whole process. The differential state variables, which are constant during the $i$-th stage, are equal to their initial values.

The particular differential state vector is characteristic for the each stage and for the $i$-th takes the form

$$
\mathbf{y}^{i}(t)=\left[\begin{array}{c}
y_{1}^{i}(t) \\
\vdots \\
y_{n_{\mathbf{y}} i}^{i}(t)
\end{array}\right] \in \mathcal{R}^{n_{\mathbf{y}^{i}}} .
$$

Therefore, the particular differential state vector for the each stage has the same size. Moreover, the particular differential state vectors can be combined together to form the differential state matrix.
Definition 3.2: The differential state matrix is formed by the particular differential state vectors is the following way

$$
\mathbf{Y}(t)=\left[\begin{array}{lll}
\mathbf{y}^{1}(t) & \cdots & \mathbf{y}^{N}(t)
\end{array}\right],
$$

where $\mathbf{y}^{i}(t), i=1, \cdots, N$ denotes the particular differential state vectors.

The properties of the differential state matrix:

1) The number of the differential state matrix rows is equal to the size of the particular differential state vector. Therefore, the number of the differential state variables can be identified.

2) The number of the differential state matrix columns is equal to the number of the process stages.

3) The differential state matrix indicates the stages, in which the chosen differential state variable has the same physical interpretation.

In the same way like the differential state matrix, the algebraic state matrix can be formed

$$
\mathbf{Z}(t)=\left[\begin{array}{lll}
\mathbf{z}^{1}(t) & \cdots & \mathbf{z}^{N}(t)
\end{array}\right]
$$

the control matrix

$$
\mathbf{U}(t)=\left[\begin{array}{lll}
\mathbf{u}^{1}(t) & \cdots & \mathbf{u}^{N}(t)
\end{array}\right],
$$

as well as the matrix of the parameters constant in the time

$$
\mathbf{P} \equiv \mathbf{p}
$$

The differential state matrix, the algebraic state matrix, the control matrix, as well as the matrix of the parameters constant in the time can be used to define the multistage descriptor process

$$
\begin{aligned}
\dot{\mathbf{Y}}(t) & =\mathbf{F}(\mathbf{Y}(t), \mathbf{Z}(t), \mathbf{U}(t), \mathbf{P}, t) \\
0 & =\mathbf{G}(\mathbf{Y}(t), \mathbf{Z}(t), \mathbf{U}(t), \mathbf{P}, t)
\end{aligned}
$$

where

$\mathbf{F}: \mathcal{R}^{n_{\mathbf{Y}}} \times \mathcal{R}^{n_{\mathbf{Z}}} \times \mathcal{R}^{n_{\mathbf{U}}} \times \mathcal{R}^{n_{\mathbf{P}}} \times \mathcal{R} \rightarrow \mathcal{R}^{n_{\mathbf{Y}}}=\mathcal{R}^{n_{\mathbf{Y}}} \times \mathcal{R}^{N}$,
$\mathbf{G}: \mathcal{R}^{n_{\mathbf{Y}}} \times \mathcal{R}^{n_{\mathbf{Z}}} \times \mathcal{R}^{n_{\mathbf{U}}} \times \mathcal{R}^{n_{\mathbf{P}}} \times \mathcal{R} \rightarrow \mathcal{R}^{n_{\mathbf{Z}}}=\mathcal{R}^{n_{\mathbf{Z}}} \times \mathcal{R}^{N}$

and $t$ denotes the duration time of the whole considered process

$$
t \in\left[\begin{array}{ll}
t_{0} & t_{F}
\end{array}\right]
$$

The generalized system description has been used to obtain the new form of the optimal control problem of three-stage chemical process .

\section{Application in the multistage process}

The matrix-based approach for the multistage DAE systems description has been applied to model of the three-reactor process (Fig. 1). The considered system is consisted of two chemical reactors and a mixing stage between them [16].

At the beginning, the first reactor is loaded with the substrate A with the volume $0.1 \mathrm{~m}^{3}$ and concentration $2000 \mathrm{~mol} / \mathrm{m}^{3}$. 
Due to reactions, which take a place in the system, the products $\mathrm{B}$ and $\mathrm{C}$ are obtained according to the scheme

$$
2 A \rightarrow B \rightarrow C \text {. }
$$

Additionally, the first reactor was equipment with a heating exchanger, which can be used to control the process temperature and in this way - to influence the trajectories of the process variables. The concentrations of the substrate and products are changing in the following way

$$
\begin{gathered}
\dot{C}_{A}=-2 k_{1}(T) C_{A}^{2} \\
\dot{C}_{B}=2 k_{1}(T) C_{A}^{2}-k_{2}(T) C_{B} \\
\dot{C}_{C}=k_{2}(T) C_{B}
\end{gathered}
$$

with the kinetics constraints

$$
k_{1}(T)=0.0444 \exp (-2500 / T)
$$

and

$$
k_{2}(T)=6889.0 \exp (-5000 / T) .
$$

Then, in the mixing stage at the time $t_{2}^{0}$, the component $\mathrm{B}$ of concentrations $C_{B}^{0}=600 \mathrm{~mol} / \mathrm{m}^{3}$ and some volume $S$ is added. Therefore, the volume and concentrations of the substrates are changing, so the following relations are satisfied

$$
\begin{gathered}
V_{2} C_{A}\left(t_{0}^{2}\right)=V_{1} C_{A}\left(t_{F}^{1}\right) \\
V_{2} C_{B}\left(t_{0}^{2}\right)=V_{1} C_{B}\left(t_{F}^{1}\right)+S C_{B}^{0} \\
V_{2} C_{C}\left(t_{0}^{2}\right)=V_{1} C_{C}\left(t_{F}^{1}\right)
\end{gathered}
$$

where $V_{1}$ is the volume of substrates loaded at the beginning of the first reactor. Therefore, the volume $V_{2}$ in the second reactor is given by

$$
V_{2}=V_{1}+S
$$

The volume $\mathrm{S}$ is a decision parameter with

$$
0 \leq S \leq 0.1\left(m^{3}\right)
$$

After the mixing stage, the substrates are loaded into the last reactor, where three reactions are taking a place

$$
\begin{gathered}
B \rightarrow D \\
B \rightarrow E \\
2 B \rightarrow F
\end{gathered}
$$

In the $2 n d$ reactor, the reactions take place under isothermal conditions. The state variables are changing in the following way

$$
\begin{gathered}
\dot{C}_{A}=0 \\
\dot{C}_{B}=-0.02 C_{B}-0.05 C_{B}-2 \times 4.0 \times 10^{-5} C_{B}^{2} \\
\dot{C}_{C}=0 \\
\dot{C}_{D}=0.02 C_{B} \\
\dot{C}_{E}=0.05 C_{B} \\
\dot{C}_{F}=4.0 \times 10^{-5} C_{B}^{2}
\end{gathered}
$$

The decision variables are the profile of the temperature $T(t)$, the duration time of the reactions in each stage, and the amount $\mathrm{S}$ of component $\mathrm{B}$, which is added at the mixing step.

The process is aimed to maximize the amount of the product $D$ at the output of the 2 nd reactor

$$
\max _{t_{1}, t_{2}, S, T(t)} V_{2} C_{D}\left(t_{2}\right)
$$

subject to the constraints on the temperature profile

$$
298 \leq T(t) \leq 398(K), \quad t^{1} \in\left[\begin{array}{ll}
t_{0}^{1} & t_{F}^{1}
\end{array}\right]
$$

According to the presented methodology, the three-stage technological process can be rewritten in the generalized matrix form. There are six state variables, which indicate the appropriate concentrations

$$
\mathbf{y}(t)=\left[\begin{array}{c}
y_{A}(t) \\
y_{B}(t) \\
y_{C}(t) \\
y_{D}(t) \\
y_{E}(t) \\
y_{F}(t)
\end{array}\right] .
$$

The particular vector of the state variables takes the form

$$
\begin{aligned}
& \mathbf{y}^{1}\left(t^{1}\right)=\left[\begin{array}{c}
y_{A}^{1}\left(t^{1}\right) \\
y_{B}^{1}\left(t^{1}\right) \\
y_{C}^{1}\left(t^{1}\right) \\
y_{D}^{1}\left(t_{0}^{1}\right) \\
y_{E}^{1}\left(t_{0}^{1}\right) \\
y_{F}^{1}\left(t_{0}^{1}\right)
\end{array}\right], \\
& \mathbf{y}^{2}\left(t^{2}\right)=\left[\begin{array}{c}
y_{A}^{2}\left(t_{0}^{2}\right) \\
y_{B}^{2}\left(t_{0}^{2}\right) \\
y_{C}^{2}\left(t_{0}^{2}\right) \\
y_{D}^{2}\left(t_{0}^{2}\right) \\
y_{E}^{2}\left(t_{0}^{2}\right) \\
y_{F}^{2}\left(t_{0}^{2}\right)
\end{array}\right], \\
& \mathbf{y}^{3}\left(t^{3}\right)=\left[\begin{array}{c}
y_{A}^{3}\left(t_{0}^{3}\right) \\
y_{B}^{3}\left(t^{3}\right) \\
y_{C}^{3}\left(t_{0}^{3}\right) \\
y_{D}^{3}\left(t^{3}\right) \\
y_{E}^{3}\left(t^{3}\right) \\
y_{F}^{3}\left(t^{3}\right)
\end{array}\right] .
\end{aligned}
$$

Therefore, the matrix of the state variables for the considered process takes the form

$$
\mathbf{Y}(t)=\left[\begin{array}{lll}
\mathbf{y}^{1}\left(t^{1}\right) & \mathbf{y}^{2}\left(t^{2}\right) & \mathbf{y}^{3}\left(t^{3}\right)
\end{array}\right] .
$$

The control function is constructed in a similar way

$$
\begin{gathered}
\mathbf{u}(t)=\left[\begin{array}{c}
T(t) \\
S \\
C_{0}
\end{array}\right] . \\
\mathbf{u}^{1}\left(t^{1}\right)=\left[\begin{array}{c}
T(t) \\
0 \\
0
\end{array}\right] .
\end{gathered}
$$




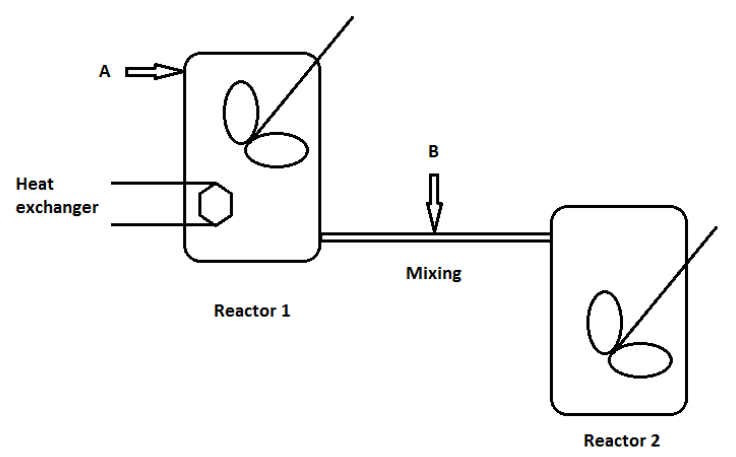

Fig. 1. The three-stage chemical process.

$$
\begin{aligned}
& \mathbf{u}^{2}\left(t^{2}\right)=\left[\begin{array}{c}
0 \\
S \\
C_{0}
\end{array}\right] . \\
& \mathbf{u}^{3}\left(t^{3}\right)=\left[\begin{array}{l}
0 \\
0 \\
0
\end{array}\right] .
\end{aligned}
$$

and finally

$$
\mathbf{U}(t)=\left[\begin{array}{lll}
\mathbf{u}^{1}\left(t^{1}\right) & \mathbf{u}^{2}\left(t^{2}\right) & \mathbf{u}^{3}\left(t^{3}\right)
\end{array}\right]
$$

\section{CONCLUSION}

In the presented article the considerations about the new generalized description approach for the multistage technological processes with the differential-algebraic constraints were discussed. Therefore, the proposed approach was characterized by some important features:

a) the general and simple schematic multistage processes description,

b) the physical interpretation of the process variables,

c) easy modification of the process by addition of new elements to the model,

d) integration of processes developed according to different standards,

e) indication the successive stage of the process by the matrix-based structure of the process variables.

From the presented reasons, the carried out theoretical considerations possess a significant practical application. Thus, the generalized description approach has been used to obtain the new form of the three-stage chemical process model.

\section{ACKNOWLEDGMENT}

This work has been supported by the National Science Center under grant: UMO-2012/07/B/ST7/01216.

\section{REFERENCES}

[1] J. R. Banga, E. Balsa-Canto, C. G. Moles, A. A. Alonso 2005. Dynamic optimization of bioprocesses: Efficient and robust numerical strategies. Journal of Biotechnology. 117:407-419, http://dx.doi.org/10.1016/j.jbiotec.2005.02.013

[2] J.T . Betts. 2010. Practical Methods for Optimal Control and Estimation Using Nonlinear Programming, Second Edition. SIAM, Philadelphia, http://dx.doi.org/10.1137/1.9780898718577

[3] L. T. Biegler. 2010. Nonlinear Programming. Concepts, Algorithms and Applications to Chemical Processes. SIAM, Philadelphia, http://dx.doi.org/10.1137/1.9780898719383

[4] L. T. Biegler. 2014. Nonlinear programming strategies for dynamic chemical process optimization. Theoretical Foundations of Chemical Engineering. 48:541-554, http://dx.doi.org/10.1134/S0040579514050157

[5] L. T. Biegler, S. Campbell, V. Mehrmann. 2012. DAEs, Control, and Optimization. Control and Optimization with Differential-Algebraic Constraints. SIAM, Philadelphia, http://dx.doi.org/10.1137/9781611972252.ch1

[6] K. E. Brenan, S.L. Campbell, L. R. Petzold. 1996. Numerical Solution of Initial- Value Problems in Differential-Algebraic Equations. SIAM, Philadelphia, http://dx.doi.org/10.1137/1.9781611971224

[7] M. Diehl, H. G. Bock, J. P. Schlöder, R. Findeisen, Z Nagy, F. Allö̈wer 2002. Real-time optimization and nonlinear model predictive control of processes governed by differential-algebraic equations. Journal of Process Control. 12:577-585, http://dx.doi.org/10.1016/S0959-1524(01)00023-3

[8] P. Drąg, K. Styczeń. 2012. A Two-Step Approach for Optimal Control of Kinetic Batch Reactor with electroneutrality condition. Przeglad Elektrotechniczny. 6/2012, pp. 176-180.

[9] S. Drozdek, U. Bazylińska. 2016. Biocompatible oil core nanocapsules as potential co-carriers of paclitaxel and fluorescent markers: preparation, characterization, and bioimaging. Colloid and Polymer Science. 294:225237, http://dx.doi.org/10.1007/s00396-015-3767-5

[10] S. Fidanova, M. Paprzycki, O. Roeva. 2014. Hybrid GA-ACO algorithm for a model parameters identification problem. Proceedings of the 2014 Federated Conference on Computer Science and Information Systems pp. 413-420, http://dx.doi.org/ 10.15439/2014F373

[11] S. Fidanova, O. Roeva. 2013. Metaheuristic techniques for optimization of an E. coli cultivation model. Biotechnology and Biotechnological Equipment. 27:3870-3876, http://dx.doi.org/10.5504/BBEQ.2012.0136

[12] P. S. Harvey Jr, H. P. Gavin, J. T. Scruggs. 2013. Optimal performance of constrained control systems. Smart Materials and Structures. 21:085001, http://dx.doi.org/10.1088/0964-1726/21/8/085001

[13] M. Kwiatkowska. 2015. DAEs method for time-varying indoor air parameters evaluation. In: A. Kotowski, K. Piekarska, B. Kaźmierczak (eds.) Interdyscyplinarne zagadnienia w inżynierii i ochronie środowiska T. 6. Wrocław 2015, pp. 214-220.

[14] M. Kwiatkowska, A. Szczurek, P. Drạg. 2016. Zastosowanie równań różniczkowo-algebraicznych do predykcji zmian parametrów powietrza wewnętrznego. Przeglad Elektrotechniczny. 5/2015, pp. 181-184, http://dx.doi.org/10.15199/48.2016.05.34

[15] K. Matyja, A. Małachowska-Jutsz, A. Mazur, K. Grabas. Assessment of toxicity using dehydrogenases activity and mathematical modeling. Ecotoxicology. 25:924-939, http://dx.doi.org/10.1007/s10646-016-1650$\mathrm{x}$.

[16] V. S Vassiliadis, R. W. H. Sargent, C. C. Pantelides. 1994. Solution of a Class of Multistage Dynamic Optimization Problems. 1. Problems without Path Constraints. Ind. Eng. Chem. Res. 33:2111-2122, http://dx.doi.org/10.1021/ie00033a014

[17] V. S Vassiliadis, R. W. H. Sargent, C. C. Pantelides. 1994. Solution of a Class of Multistage Dynamic Optimization Problems. 2. Problems with Path Constraints. Ind. Eng. Chem. Res. 33: 2123-2133, http://dx.doi.org/10.1021/ie00033a015

[18] Z.-H. Yang, W.-J. Cui, Y. Tang. 2008. Optimal control with DAE constraints. International Conference on Industrial Engineering and Engineering Management, 2008. pp. 188-192, http://dx.doi.org/10.1109/IEEM.2008.4737857

[19] Z.-H. Yang, F. Guo. 2012. Optimal Control Conditions with DifferentialAlgebraic Equation Constraints. Advanced Science Letters. 6:654-659, http://dx.doi.org/10.1166/asl.2012.2300 